\title{
SUBSTÂNCIAS HÚMICAS DE TURFA: ESTUDO DOS PARÂMETROS QUE INFLUENCIAM NO PROCESSO DE EXTRAÇÃO ALCALINA
}

\author{
André Henrique Rosa, Julio Cesar Rocha*, Maysa Furlan \\ Instituto de Química de Araraquara - UNESP - CP 355 - 14800-900 - Araraquara - SP \\ Recebido em 17/6/99; aceito em 28/11/99
}

\begin{abstract}
HUMIC SUBSTANCES OF PEAT: STUDY OF THE PARAMETERS THAT INFLUENCE ON THE PROCESS OF ALKALINE E EXTRACTION. This study reports the parameters which influence on extraction of humic substances (HS) from peat. The yield, organic and inorganic contend, $E_{4} /$ $\mathrm{E}_{6}$ ratio and elemental composition $(\mathrm{C}, \mathrm{H}, \mathrm{N})$ of the extracted $\mathrm{HS}$ have been determined. The functional groups were identified by Fourier-transform infrared espectroscopy. The results showed influence of the extractor type, concentration, time, temperature and granular texture of the sample in the extraction. This work contributes to better understanding of the extraction process showing the necessity of the standard method to extract humic substances from soil.
\end{abstract}

Keywords: humic substances; humic acid; fulvic acid; peat; extraction.

\section{INTRODUÇÃOO}

Substâncias húmicas (SH) são formadas pela transformação de biomoléculas, durante o processo de decomposição de resíduos vegetais e animais presentes no ambiente ${ }^{1,2}$. Devido a natureza heterogênea e complexa das $\mathrm{SH}$ pouco se sabe sobre sua estrutura química e apresentam-se como moléculas polidifusas com elevada massa molar ${ }^{3,4}$. Possuem alto teor de grupos funcionais contendo oxigênio na forma de carboxilas, hidroxilas fenólicas e carbonilas ${ }^{5}$. Operacionalmente as SH são fracionadas em função de sua solubilidade a diferentes valores de $\mathrm{pH}$ em: ácidos húmicos )m ), ácidos fúlvicos (AF) e humina.

Devido suas características estruturais as $\mathrm{SH}$ podem interagir com metais ${ }^{6,7}$ e compostos orgânicos como por exemplo, pesticidas e herbicidas presentes no ambiente ${ }^{8-10}$. Para melhor compreender o comportamento das SH, estas têm sido estudadas sob diferentes aspectos: caracterização de estruturas parciais ${ }^{11}$, determinação de constantes de equilíbrio de espécies metal- $\mathrm{SH}^{12}$, labilidade relativa de metais ${ }^{13}$ etc.. Entretanto, a primeira etapa para esses estudos é a extração das SH. Das condições experimentais utilizadas no procedimento de extração dependerão as futuras interpretações. Assim, é importante o desenvolvimento de métodos analíticos que permitam extrair $\mathrm{SH}$ de solos, com mínimas alterações nas estruturas originais, em tempo relativamente curto, elevado rendimento e baixo teor de contaminantes ${ }^{14}$.

Ainda não existe uma metodologia para extração de SH de solos, oficialmente adotada. Alguns autores têm utilizado extratores mais brandos como pirofosfato de sódio ${ }^{15}$, agentes complexantes $^{16}$, ácido fórmico ${ }^{17}$, misturas ácidas ${ }^{18}$ e solventes orgânicos ${ }^{19}$. Mesmo havendo risco de alterações estruturais, outros autores preferem a extração mais completa das SH utilizando álcalis ${ }^{1,2}$. A International Humic Substances Society (IHSS) recomenda um procedimento baseado em 4 horas de extração com solução de $\mathrm{NaOH} 0,1 \mathrm{~mol} / \mathrm{L}$ à temperatura ambiente na razão solo/extrator $1: 10(\mathrm{~m} / \mathrm{v})$ sob atmosfera de nitrogênio $^{20}$. Entretanto, de acordo com Parsons ${ }^{21}$, vários fatores influenciam no procedimento de extração e muitas questões ainda estão por ser respondidas.

e-mail: jrocha@iq.unesp.br

\#Dedicated to the $60^{\text {th }}$ birthday of Dr. Peter Burba and Prof. Dr. Celso A. F. Graner
Neste trabalho estudaram-se os parâmetros que influenciam na extração alcalina de SH de turfa como, tipo de extrator alcalino, concentração, tempo, temperatura de extração, razão turfa/extrator e granulometria da amostra.

\section{MATERIAIS E MÉTODOS}

\section{Reagentes e soluções}

Utilizou-se água previamente destilada e purificada em sistema deionizador de água, Milli-Qplus até apresentar resistividade $18,2 \mathrm{M} \mathrm{ohm} / \mathrm{cm}$ a $25^{\circ} \mathrm{C}$ e reagentes de grau analítico.

\section{Coleta e preparo da amostra}

Coletou-se amostra de turfa $(30 \mathrm{~cm}$ de profundidade $) \mathrm{em}$ área localizada no município de Ribeirão Preto-SP. Após secagem ao ar, triturou-se em grál de porcelana e peneirouse a $2 \mathrm{~mm}$.

\section{Tipo, concentração do extrator e tempo de extração}

Fizeram-se extrações das SH variando-se as soluções extratoras $(\mathrm{NaOH}$ e $\mathrm{KOH})$ e suas respectivas concentrações $(0,1,0,5$ e $1,0 \mathrm{~mol} / \mathrm{L})$, por 4 horas, razão turfa/extrator $1: 10$ $(\mathrm{m} / \mathrm{v})$, temperatura ambiente $\left(25-30^{\circ} \mathrm{C}\right)$, sob atmosfera de ni$\operatorname{trogênio}^{22}$ e agitação mecânica. Após extração, centrifugouse a 2000.g por 10 minutos para separar a humina, fração insolúvel. Concentrou-se o extrato húmico $(50 \mathrm{~mL})$ em evaporador rotativo a $55^{\circ} \mathrm{C}$ até cerca de $10 \mathrm{~mL}$, transferiu-se para placa de Petri e secou-se em estufa com circulação de ar. Repetiu-se esse procedimento variando-se o tempo de extração entre 1-8 horas.

\section{Razão turfa/extrator, temperatura de extração e granulometria da amostra}

Fizeram-se extrações das SH utilizando-se solução de KOH $0,5 \mathrm{~mol} / \mathrm{L}$, por 4 horas à temperatura ambiente, sob atmosfera de nitrogênio e agitação mecânica com diferentes razões turfa/ extrator: $1: 5,1: 10,1: 20,1: 30,1: 40$ e 1:50 (m/v). Posteriormente, com razão turfa/extrator 1:20 (m/v), estudaram-se a influência da temperatura de extração $\left(25,50\right.$ e $\left.75^{\circ} \mathrm{C}\right)$ e granulometria da turfa $(0,21,0,59$ e $1,0 \mathrm{~mm})$. 
Fracionamento das substâncias húmicas em ácidos húmicos e fúlvicos

Para fracionamento em ácidos húmicos e fúlvicos, ajustou-se o $\mathrm{pH}$ das $\mathrm{SH}$ em 1,5 com solução de $\mathrm{HCl}$ 6,0 mol/L. Após decantação e centrifugação a $2000 . g$ por 10 minutos o AH foi transferido para placa de Petri e seco em estufa de circulação de ar a $55^{\circ} \mathrm{C}$. O AF foi concentrado em evaporador rotativo a $55^{\circ} \mathrm{C}$, transferido para placa de Petri e seco em estufa de circulação de ar.

\section{Teor de matéria orgânica e rendimento das extrações}

Determinou-se o teor de matéria orgânica pela diferença entre a massa de material seco submetido à calcinação $\left(750^{\circ} \mathrm{C}\right.$ por 4 horas) e o resíduo final obtido ${ }^{23}$. O rendimento da extração foi obtido pela diferença entre a massa de turfa seca submetida à extração e a massa de SH extraída. Calcularam-se os rendimentos de $\mathrm{AH}$ e de $\mathrm{AF}$ pelas diferenças entre a massa de SH submetida à acidificação a $\mathrm{pH} 1,5$ e as massas obtidas para os respectivos ácidos.

\section{Espectroscopia na região do visível e do infravermelho}

Após purificação das amostras por diálise, conforme procedimento descrito por Town et al. ${ }^{24}$, dissolveram-se $0,02 \mathrm{~g}$ de $\mathrm{AH}$ e de $\mathrm{AF}$ em $10 \mathrm{~mL}$ de solução de $\mathrm{NaHCO}_{3} \quad 0,05 \mathrm{~mol} / \mathrm{L}$ e determinaram-se as razões entre as absorbâncias lidas a 465 e $665 \mathrm{~nm}\left(\mathrm{E}_{4} / \mathrm{E}_{6}\right)^{25}$. Obtiveram-se espectros na região do infravermelho com pastilhas de $\mathrm{KBr}(200 \mathrm{mg})$ e $0,5 \mathrm{mg}$ de cada ácido, submetendo-se a mistura (KBr: $\mathrm{AH}$ ou $\mathrm{AF})$ à pressão de $10 \mathrm{t} / \mathrm{cm}^{2}$ em pastilhador de $14 \mathrm{~mm}$ de diâmetro.

\section{RESULTADOS E DISCUSSÃO}

\section{Tipo e concentração do extrator}

A Figura 1 mostra resultados referentes ao rendimento, teor de matéria orgânica e inorgânica das SH extraídas em função da concentração e do tipo de extrator. De acordo com Clapp et al. ${ }^{20}$ e Swift ${ }^{26}$ o processo de extração alcalina compreende a ionização dos grupos ácidos, causando expansão e repulsão de cargas da macromolécula húmica resultando na transferência das SH da fase sólida para a solução. Conseqüentemente, a solubilidade das SH é dependente do $\mathrm{pH}$, força iônica e natureza dos eletrólitos ${ }^{24}$.

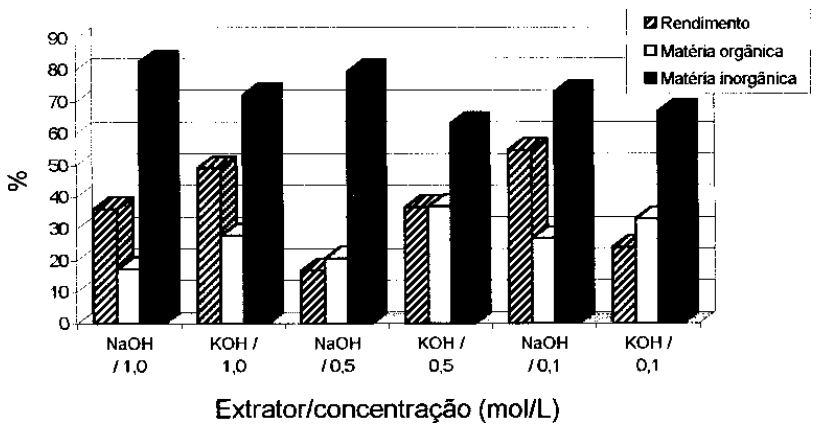

Figura 1. Influência do tipo e da concentração da solução extratora no rendimento, teor de matéria orgânica e inorgânica das substâncias húmicas extraídas de turfa.

No intervalo de concentração estudado, o rendimento das SH extraídas aumentou em função do aumento da concentração da solução de KOH. Entretanto, este comportamento não foi observado quando utiliza-se solução de $\mathrm{NaOH}$ pois, neste caso, o menor rendimento foi caracterizado utilizando-se concentração intermediária, ou seja $0,5 \mathrm{~mol} / \mathrm{L}$. Provavelmente, as diferentes concentrações e características termodinâmicas (raio de hidratação ${ }^{27}$ e mobilidade iônica ${ }^{28}$ ) dos contra íons $\mathrm{Na}^{+} \mathrm{e} \mathrm{K}^{+}$influenciaram no processo de solvatação dos grupos ionizados, e conse- qüentemente, no rendimento da extração. Com soluções extratoras mais diluídas $(0,1 \mathrm{~mol} / \mathrm{L})$ utilizando-se $\mathrm{NaOH}$ o rendimento é cerca de duas vezes maior. Entretanto, em concentrações mais elevadas $(1,0$ e $0,5 \mathrm{~mol} / \mathrm{L})$ o rendimento é maior quando se utiliza solução de $\mathrm{KOH}$ e, além disso, o material inorgânico co-extraído é menor. A vantagem de extrair SH com baixo teor de cinzas é diminuir etapas de purificação as quais sempre causam perda de material e possibilitam modificações estruturais nas SH. Assim, nesse trabalho, optou-se pela não purificação das $\mathrm{SH}$ extraídas para melhor avaliação dos parâmetros envolvidos no processo de extração. Entretanto, a não purificação das SH tornou inviável a comparação entre os resultados de caracterização obtidos com dados da literatura para $\mathrm{SH}$ com baixos teores de cinzas.

A razão $\mathrm{E}_{4} / \mathrm{E}_{6}$ está diretamente relacionada à condensação estrutural, sendo indicativo do grau de humificação, aromaticidade, peso molecular e conteúdo ácido das SH. A diminuição dos valores da razão $\mathrm{E}_{4} / \mathrm{E}_{6}$ indicam maior condensação enquanto que maiores razões estão associadas a estruturas menos condensadas 1,4 .

A Tabela 1 mostra resultados referentes à razão das absorbâncias $\mathrm{E}_{4} / \mathrm{E}_{6}$ das $\mathrm{SH}$, AH e AF extraídos de turfa utilizandose soluções extratoras de hidróxido de sódio e de potássio em diferentes concentrações.

Tabela 1. Influência do tipo e concentração da solução extratora na razão das absorbâncias $\mathrm{E}_{4} / \mathrm{E}_{6}$, das substâncias húmicas, ácidos húmico e fúlvico extraídos de turfa.

\begin{tabular}{ccccc}
\hline Solução & $\begin{array}{c}\text { Concentração } \\
\text { extratora }\end{array}$ & \multicolumn{3}{c}{$\mathrm{E}_{4} / \mathrm{E}_{6}$} \\
\cline { 3 - 5 } & (mol/L) & $\mathrm{SH}$ & $\mathrm{AH}$ & $\mathrm{AF}$ \\
$\mathrm{NaOH}$ & 1,0 & 5,75 & 4,22 & 4,05 \\
$\mathrm{NaOH}$ & 0,5 & 5,21 & 4,65 & 4,20 \\
$\mathrm{NaOH}$ & 0,1 & 3,75 & 4,72 & 4,63 \\
$\mathrm{KOH}$ & 1,0 & 3,14 & 4,20 & 3,67 \\
$\mathrm{KOH}$ & 0,5 & 3,55 & 3,94 & 3,02 \\
$\mathrm{KOH}$ & 0,1 & 3,58 & 3,80 & 3,26 \\
\hline
\end{tabular}

A maior tendência do AF em formar pontes de hidrogênio intra e intermolecular ${ }^{29}$ é responsável pelas menores razões $\mathrm{E}_{4} /$ $\mathrm{E}_{6}$ se comparadas àquelas referentes ao $\mathrm{AH}$. Independentemente da concentração, as $\mathrm{SH}$ extraídas com solução de $\mathrm{KOH}$, tiveram menores razões $\mathrm{E}_{4} / \mathrm{E}_{6}$ indicando maior condensação estrutural.

Os resultados da Tabela 2 também indicam a influência do tipo e concentração do extrator utilizado na composição elementar das substâncias húmicas extraídas. Os valores das razões molares $\mathrm{H} / \mathrm{C}$ estão diretamente relacionados ao conteúdo ácido das SH e observa-se pela Tabela 2 que a utilização de $\mathrm{KOH}$ e soluções extratoras mais diluídas extraem SH com maior acidez. Esses resultados estão associados ao processo de neutralização e contrabalanço das cargas das SH pelo extrator $^{20,26}$. A diferença entre os teores de nitrogênio das $\mathrm{SH}$ extraídas pode indicar uma possível contaminação de substâncias de natureza não húmica como por exemplo, proteínas e aminoácidos quando a extração é feita com $\mathrm{NaOH}$ ou sugerir uma extração incompleta do material húmico por $\mathrm{KOH}$.

A Figura 2 mostra forte influência do tipo e da concentração da solução extratora no teor de $\mathrm{AH}$ e $\mathrm{AF}$ extraídos da amostra de turfa. Diminuindo a concentração do álcali, a extração do AH aumenta e a do AF reduz. Essas evidências ficaram caracterizadas inclusive por visualização da diferença de coloração (castanho, amarelo e quase incolor) da fração fúlvica extraída em função da concentração da solução extratora utilizada. Isto decorre do aumento da concentração do álcali causando rompimento de forças intermoleculares que ligam o $\mathrm{AF}$ ao $\mathrm{AH}$, como pontes de hidrogênio ou ligações do tipo éster ${ }^{1}$. Comportamento semelhante é observado na utilização de pirofosfato de sódio como solução extratora ${ }^{30}$. 
Tabela 2. Resultados da composição elementar das substâncias húmicas extraídas de turfa.

\begin{tabular}{ccccc}
\hline $\begin{array}{c}\text { Substância } \\
\text { húmica* }\end{array}$ & $\mathrm{C}$ & $\mathrm{H}$ & $\mathrm{N}$ & $\mathrm{H} / \mathrm{C}$ \\
\hline $\mathbf{S H}_{\mathbf{A}}$ & 27,59 & 4,44 & 1,52 & 1,94 \\
$\mathbf{S H}_{\mathbf{B}}$ & 26,88 & 4,73 & 1,43 & 2,11 \\
$\mathbf{S H}_{\mathbf{C}}$ & 22,31 & 5,18 & 1,58 & 2,78 \\
$\mathbf{S H}_{\mathbf{D}}$ & 26,19 & 5,48 & 0,71 & 2,51 \\
$\mathbf{S H}_{\mathbf{E}}$ & 25,79 & 6,13 & 0,57 & 2,85 \\
$\mathbf{S H}_{\mathbf{F}}$ & 23,06 & 6,35 & 0,49 & 3,31 \\
\hline
\end{tabular}

$\mathbf{S H}_{\mathbf{A}}=$ extração com solução de $\mathrm{NaOH} 1,0 \mathrm{~mol} / \mathrm{L}$;

$\mathbf{S H}_{\mathbf{B}}=$ extração com solução de $\mathrm{NaOH} 0,5 \mathrm{~mol} / \mathrm{L}$;

$\mathbf{S H}_{\mathbf{C}}=$ extração com solução de $\mathrm{NaOH} 0,1 \mathrm{~mol} / \mathrm{L}$;

$\mathbf{S H}_{\mathbf{D}}=$ extração com solução de $\mathrm{KOH} 1,0 \mathrm{~mol} / \mathrm{L}$;

$\mathbf{S H}_{\mathbf{E}}=$ extração com solução de $\mathrm{KOH} 0,5 \mathrm{~mol} / \mathrm{L}$;

$\mathbf{S H}_{\mathbf{F}}=$ extração com solução de $\mathrm{KOH} 0,1 \mathrm{~mol} / \mathrm{L}$;

* substância húmica não purificada

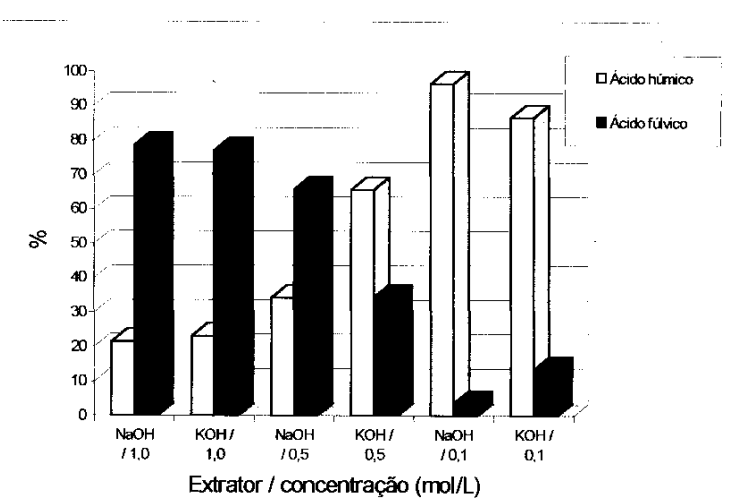

Figura 2. Influência da solução extratora e da sua concentração no teor de $A H$ e AF extraído de turfa.

As bandas de absorção dos espectros na região do infravermelho obtidos dos ácidos húmicos e fúlvicos extraídos com soluções de $\mathrm{NaOH}$ e $\mathrm{KOH}$ em diferentes concentrações são compatíveis com aquelas encontradas na literatura ${ }^{3,31}$. As principais bandas de absorção do espectros de $\mathrm{AH}$ são listadas na Tabela 3 e mostradas na Figura 3.

Os espectros dos AF (Figura 4) mostram praticamente as mesmas frequências de absorção, com excessão da banda cerca de $1700 \mathrm{~cm}^{-1}$ que encontra-se deslocada para frequências próximas de $1680 \mathrm{~cm}^{-1}$ podendo estar associada ao estiramento $\mathrm{C}=\mathrm{O}$ de cetonas conjugadas. Também verifica-se nos espectros do AF o desaparecimento da banda na região de $3700 \mathrm{~cm}^{-1}$ associada ao estiramento $\mathrm{OH}$ de silicatos e o fortalecimento da banda na região de $1000 \mathrm{~cm}^{-1}$ característica marcante de estiramento $\mathrm{C}-\mathrm{O}$ de carboidratos, indicando assim, ser este o contaminante principal da fração fúlvica.

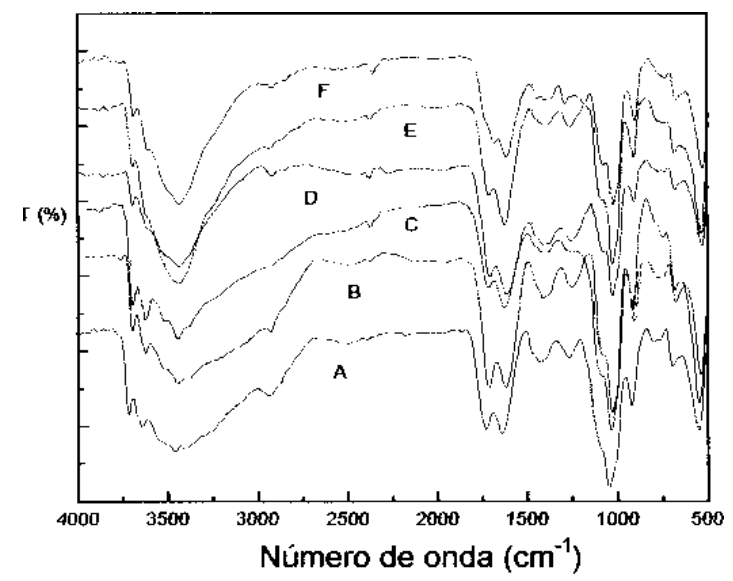

Figura 3. Espectros no IV de ácidos húmicos $(p H: 1,5)$ extraídos de turfa com soluções de $\mathrm{NaOH}$ e $\mathrm{KOH}$ em diferentes concentrações. (A: $\mathrm{NaOH} \mathrm{1,0} \mathrm{mol/L;} \mathrm{B:} \mathrm{KOH} \mathrm{1,0} \mathrm{mol/L;} \mathrm{C:} \mathrm{NaOH} \mathrm{0,5} \mathrm{mol/L;} \mathrm{D:} \mathrm{KOH}$ 0,5 mol/L; E: NaOH 0,1 mol/L; F: KOH 0,1 mol/L).

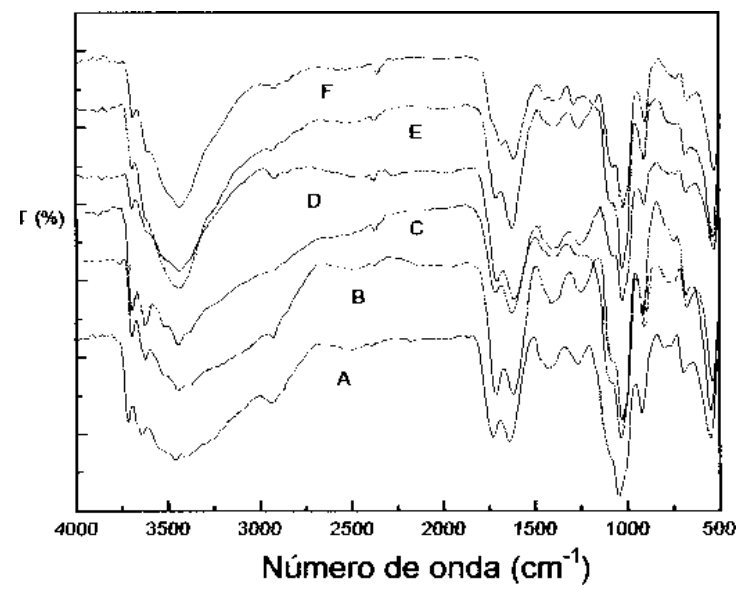

Figura 4. Espectros no IV de ácidos fúlvicos $(p H: 1,5)$ extraídos de turfa com soluções de $\mathrm{NaOH}$ e $\mathrm{KOH}$ em diferentes concentrações. (A: $\mathrm{NaOH} \mathrm{1,0} \mathrm{mol/L;} \mathrm{B:} \mathrm{KOH} \mathrm{1,0} \mathrm{mol/L;} \mathrm{C:} \mathrm{NaOH} \mathrm{0,5} \mathrm{mol/L;} \mathrm{D:} \mathrm{KOH}$ 0,5 mol/L; E: NaOH 0,1 mol/L; F: $\mathrm{KOH} \mathrm{0,1} \mathrm{mol/L).}$

Comparando-se os espectros dos ácidos húmicos e fúlvicos extraídos com solução de $\mathrm{KOH}$ e de $\mathrm{NaOH}$ em diferentes concentrações (Figuras 3 e 4), não se observam alterações significativas quanto à natureza dos grupamentos presentes na estrutura das macromoléculas húmicas.

\section{Tempo de extração e razão turfa/extrator}

A Figura 5 mostra a influência do tempo de extração no teor de matéria orgânica extraída. Segundo Stevenson ${ }^{1}$, a quantidade

Tabela 3. Bandas de absorção na região do infravermelho observadas nos espectros de ácidos húmicos extraídos de turfa utilizando-se soluções extratoras de $\mathrm{NaOH}$ e $\mathrm{KOH}$ em diferentes concentrações.

\begin{tabular}{|c|c|}
\hline Número de onda $\left(\mathrm{cm}^{-1}\right)$ & Atribuição \\
\hline $3700-3750$ & estiramento $\mathrm{OH}$ de silicatos; \\
\hline$\sim 3400$ & estiramento $\mathrm{OH}$ de álcoois e/ou fenóis e/ou ácidos carboxílicos e/ou NH; \\
\hline $2.940-2.900$ & estiramento assimétrico de $\mathrm{C}-\mathrm{H}$ de alifáticos $\left(-\mathrm{CH}_{2}\right)$ \\
\hline 1720 & estiramento $\mathrm{C}=\mathrm{O}$, de grupos carboxílicos e/ou carbonílicos; \\
\hline $1.620-1.600$ & estiramento $\mathrm{C}=\mathrm{C}$ de anel aromático; \\
\hline $\begin{array}{l}1.590-1.517 \\
1450\end{array}$ & $\begin{array}{l}\text { estiramento } \mathrm{C}=\mathrm{C} \text { de anel aromático, deformação } \mathrm{N}-\mathrm{H} \text { de aminoácidos e estiramento } \mathrm{C}=\mathrm{N} \text {; } \\
\text { deformacão } \mathrm{CH} \text { de alifáticos }\left(-\mathrm{CH}_{2} ;-\mathrm{CH}_{3}\right) \text {; }\end{array}$ \\
\hline $1.400-1.370$ & deformação O-H, deformação C-H $\left(-\mathrm{CH}_{2} ;-\mathrm{CH}_{3}\right)$, estiramento simétrico do $\mathrm{COO}^{-}$ \\
\hline $1.170-950$ & estiramento $\mathrm{C}-\mathrm{O}$ de álcoois e/ou fenóis e/ou carboidratos, impurezas de silicatos $(\mathrm{Si}-\mathrm{O})$; \\
\hline$\sim 900$ & deformações fora do plano de $\mathrm{CH}$ de aromáticos \\
\hline
\end{tabular}


de matéria orgânica (MO) extraída com solventes alcalinos elevase com o tempo de extração devido à lenta despolimerização de complexos de alto peso molecular. Esses complexos fazem parte da humina e estão associados a sesquióxidos e silicatos. Assim, o tempo necessário para extração depende do grau de humificação da MO e das características do material inorgânico presente na matriz. Na amostra utilizada neste estudo, verifica-se a despolimerização da MO presente na humina até 240 minutos, após esse tempo não há aumento considerável no teor de MO extraída.

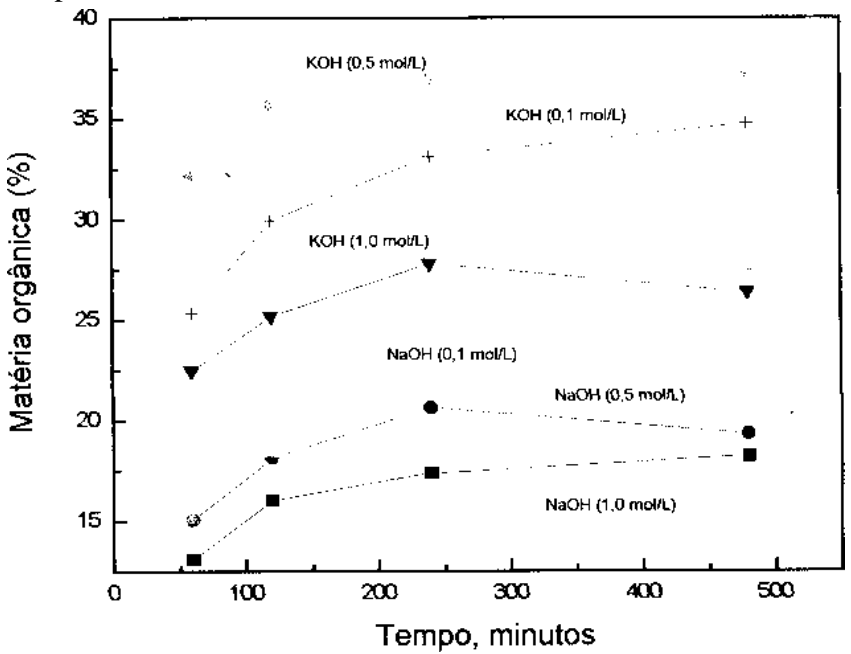

Figura 5. Influência do tempo de extração no teor de matéria orgânica extraída de turfa.

Utilizando-se a razão turfa/extrator 1:20 (m/v) o rendimento de material orgânico extraído foi maior. Em razões maiores observou-se apenas aumento do teor de material inorgânico mas não no conteúdo de material orgânico extraído. A variação do tempo de extração e das razões turfa/extrator não apresentaram diferenças significativas nos valores da razão $\mathrm{E}_{4} / \mathrm{E}_{6}$ das $\mathrm{SH}$ extraídas.

\section{Temperatura de extração}

A Tabela 4 lista resultados da razão $\mathrm{E}_{4} / \mathrm{E}_{6}$ e teores de $\mathrm{AH}$ e AF extraídos em função da temperatura de extração. O aumento da temperatura não exerceu grande influência no rendimento do material extraído. Entretanto, há redução do teor de $\mathrm{AH}$ com o aumento da temperatura provavelmente devido ao rompimento de ligações tipo pontes de hidrogênio ou éster presentes na estrutura das moléculas húmicas ${ }^{32}$.

Tabela 4. Influência da temperatura $\left(25,50\right.$ e $\left.75^{\circ} \mathrm{C}\right)$ na extração das SH de turfa.

\begin{tabular}{cccccc}
\hline $\begin{array}{c}\text { Temperatura de } \\
\text { extração }\left({ }^{\circ} \mathrm{C}\right)\end{array}$ & $\mathrm{SH}$ & \multicolumn{2}{c}{$\mathrm{AH}$} & \multicolumn{2}{c}{$\mathrm{AF}$} \\
\hline 25 & 3,87 & 22,87 & 3,94 & 77,13 & 3,02 \\
50 & 3,35 & 20,43 & 4,10 & 79,57 & 3,22 \\
75 & 3,62 & 16,18 & 4,56 & 83,82 & 3,35 \\
\hline
\end{tabular}

$\mathrm{R}$ - rendimento $(\%)$

Comparando-se os espectros de $\mathrm{AH}$ extraídos a 50 e $75^{\circ} \mathrm{C}$, com o espectro de $\mathrm{AH}$ extraído a $25^{\circ} \mathrm{C}$ (Figura 6) verifica-se o desaparecimento das bandas em torno de $3750 \mathrm{~cm}^{-1}$ (estiramento $\mathrm{OH}$ de silicatos), $3450 \mathrm{~cm}^{-1}$ (estiramento $\mathrm{OH}$ de álcoois e/ou fenóis) e $1030 \mathrm{~cm}^{-1}$ (estiramento $\mathrm{CO}$ de álcoois e/ou fenóis e $\mathrm{SiO}$ de impurezas de silicatos). Essas diferenças sugerem que a elevação da temperatura de extração pode causar oxidação dos grupos hidroxílicos a carbonílicos de cetonas causando o rompimento do complexo $\mathrm{AH}$-argila, havendo assim, liberação de impurezas de silicatos agregados à estrutura do complexo para a fração insolúvel (humina).

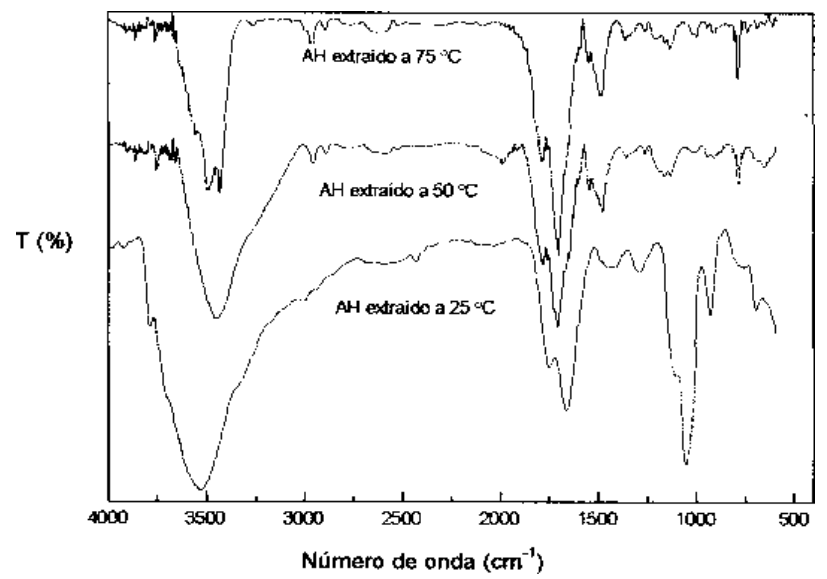

Figura 6. Espectros no IV de ácidos húmicos $(p H=1,5)$ extraídos de turfa em diferentes temperaturas $\left(25,50\right.$ e $\left.75^{\circ} \mathrm{C}\right)$.

As diferenças observadas nos espectros de ácidos fúlvicos extraídos a 50 e $75^{\circ} \mathrm{C}$ em relação ao espectro de $\mathrm{AF}$ extraído a $25^{\circ} \mathrm{C}$ (Figura 7) foram: alargamento da banda em 3600 $3000 \mathrm{~cm}^{-1}$ (estiramento $\mathrm{OH}$ de álcoois e/ou fenóis e/ou ácidos carboxílicos), aparecimento de banda em $1720 \mathrm{~cm}^{-1}$ (estiramento $\mathrm{C}=\mathrm{O}$ de ácidos carboxílicos) e deslocamento/diminuição da banda referente ao estiramento $\mathrm{C}-\mathrm{O}$ para região de $1100 \mathrm{~cm}^{-1}$. Essas alterações no espectro possivelmente indicam a conversão de grupamentos hidroxílicos à ácidos carboxílicos devido oxidação durante o processo de extração. A diminuição da banda na região de $1000 \mathrm{~cm}^{-1}$ referente ao estiramento $\mathrm{C}-\mathrm{O}$ também pode estar associada a degradação com a elevação da temperatura de carboidratos presentes na fração fúlvica.

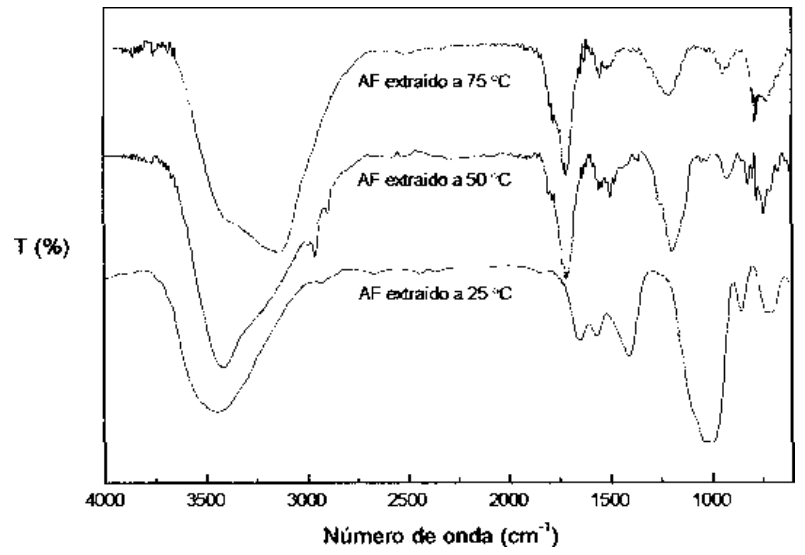

Figura 7. Espectros no IV de ácidos fúlvicos $(p H=1,5)$ extraídos de turfa em diferentes temperaturas $\left(25,50\right.$ e $\left.75^{\circ} \mathrm{C}\right)$.

$\mathrm{O}$ aumento dos valores da razão $\mathrm{E}_{4} / \mathrm{E}_{6}$ (Tabela 4) de $\mathrm{AH}$ e AF também mostram diferenças que inferem a redução da condensação e do peso molecular, em função da elevação da temperatura de extração. Assim, os valores da razão $\mathrm{E}_{4} / \mathrm{E}_{6}$ e a análise dos espectros obtidos na região do infravermelho mostram que a elevação da temperatura da extração causa alterações estruturais tanto no AH como no AF. Essas modificações foram discutidas com base na comparação dos espectros de infravermelho e visível e estão de acordo com Pang, et al. ${ }^{33}$ os quais utilizando $\mathrm{RMN}{ }^{1} \mathrm{H}$ e ${ }^{13} \mathrm{C}$ verificaram que o aumento da temperatura durante a extração exerce influência nas características estruturais do material extraído pelo favorecimento de processos oxidativos. 


\section{Influência da granulometria do solo}

A Tabela 5 lista os resultados referentes ao estudo da granulometria da turfa submetida a extração. $\mathrm{O}$ rendimento bruto da extração praticamente não variou com a granulometria da amostra de turfa. Entretanto, os teores de material orgânico e inorgânico extraídos são diretamente influenciados pela granulometria da amostra. Ou seja, menor granulometria favorece a extração de maior teor de matéria orgânica e, conseqüentemente, diminui o teor de matéria inorgânica (MI) devido ao aumento da superfície de contato da turfa com a solução extratora.

Tabela 5. Influência da granulometria $(0,21 ; 0,59$ e $1,00 \mathrm{~mm})$ da turfa na extração das SH.

\begin{tabular}{cccc}
\hline Granulometria da & \multicolumn{3}{c}{ Substâncias húmicas } \\
turfa (mm) & R $(\%)$ & MO $(\%)$ & MI $(\%)$ \\
\hline 0,21 & 49,67 & 31,34 & 68,66 \\
0,59 & 52,18 & 28,59 & 71,41 \\
1,00 & 50,35 & 25,43 & 74,57 \\
\hline
\end{tabular}

$\mathrm{R}=$ rendimento $(\%)$

$\mathrm{MO}=$ matéria orgânica $(\%)$;

$\mathrm{MI}=$ matéria inorgânica $(\%)$;

\section{CONCLUSÕES}

Os resultados mostraram influência do tipo de extrator alcalino $(\mathrm{NaOH}$ e $\mathrm{KOH})$, concentração $(1,0,0,5$ e $0,1 \mathrm{~mol} / \mathrm{L})$, tempo de extração $(60,120,240$ e 480 minutos $)$, temperatura de extração $\left(25,50\right.$ e $\left.75^{\circ} \mathrm{C}\right)$, e granulometria da turfa $(0,21 ; 0,59$ e $1,0 \mathrm{~mm})$ nas características estruturais, rendimento e teor de cinzas das $\mathrm{SH}$ extraídas de amostra de turfa. $\mathrm{O}$ extrator alcalino e sua concentração influenciaram no rendimento, teor de cinzas, razão $\mathrm{E}_{4} / \mathrm{E}_{6}$, e teor de $\mathrm{AH}$ e $\mathrm{AF}$ extraídos, mas não na presença dos grupos funcionais presentes na estrutura dos ácidos. O teor de matéria orgânica extraída (solúvel em álcali) eleva-se até 240 minutos e o rendimento foi maior utilizando-se a razão turfa/extrator $1: 20(\mathrm{~m} / \mathrm{v})$. Entretanto, estes parâmetros não influenciaram nas razões de absorbâncias $\left(\mathrm{E}_{4} / \mathrm{E}_{6}\right)$ das $\mathrm{SH}$ extraídas. Altas temperaturas causam alterações estruturais nos ácidos devido à oxidação e quebra do complexo húmico-silicato e, aumentando o teor de AF em detrimento do teor de AH. A diminuição da granulometria da turfa permite a elevação do teor de matéria orgânica extraída devido ao aumento de sua superfície de contato com o solvente extrator.

As melhores condições para extração das substâncias húmicas da amostra de turfa em estudo foram: solução extratora $\mathrm{KOH}$ (0,5 mol/L), tempo de extração 4 horas, razão turfa/extrator 1:20 $(\mathrm{m} / \mathrm{v})$, temperatura ambiente $\left(25-30^{\circ} \mathrm{C}\right)$, atmosfera de nitrogênio, agitação mecânica e granulometria de $0,21 \mathrm{~mm}$. A solução extratora $\mathrm{KOH}(0,5 \mathrm{~mol} / \mathrm{L})$ apresentou vantagens em relação ao $\mathrm{NaOH}$, para a amostra estudada, devido ao maior rendimento e redução de contaminantes no processo de extração.

Este trabalho contribui para um melhor entendimento dos parâmetros relacionados com o processo de extração alcalina de SH e caracteriza a importância da sistematização do processo de extração para melhor comparação entre resultados de amostras com características químicas distintas coletadas em diferentes localidades.

\section{AGRADECIMENTOS}

Os autores agradecem a FAPESP (Proc. nœ 95/4682-9 e 97/ 10673-8) e CNPq por bolsas e suporte financeiro concedido.

\section{REFERÊNCIAS}

1. Stevenson, F. J.; Humus Chemistry. Wiley \& Sons; New York, 1982; p.198.
2. Senesi, N.; Miano, T. M.; Humic substances in the global environmental and implications on human health. Elsevier, Amsterdan, 1994.

3. Swift, R. S.; In: Methods of soil analysis. Part 3. chemical methods - SSSA Book series no 5". Soil Science Society of America and American Society of Agronomy, Madison, 1996, p. 1011.

4. Stevenson, F. J.; In: Humic Substances in soil, sediment and water; Aiken, G. R.; Mcknight, D. M.; Wershaw, R. L., Eds. Wiley \& Sons; New York, 1985; p.13.

5. Jordão, C. P.; Costa, E. D.; Brune, W.; Gourlart, A. T.; Quim. Nova 1993, 16, 517.

6. Burba, P.; Rocha, J. C.; Klockow, D.; Fresenius J. Anal. Chem. 1994, 349, 800.

7. Rocha, J. C.; Sene, J. J.; Burba, P.; Klockow, D.; J. Braz. Chem. Soc. 1998, 9, 79.

8. Landgraf, M. D.; Silva, S. C.; Resende, M. O. O.; Anal. Chim. Acta. 1998, 368, 155.

9. Santos, T. C.; Rocha, J. C.; Alonso, R. M.; Martìnez, E.; Ibanez, C.; Barceló, D.; Environ. Sci. Technol. 1998, 32, 3479.

10. Martin, L. N.; Vieira, E. M.; Sposito, G.; Environ. Sci. Technol. 1994, 28, 1867.

11. Chiavari, G.; Torsi, G.; Fabbri, D.; Galletti, G. C.; Analyst 1994, 119, 1141.

12. Pinheiro, J. P.; Mota, A. M.; Gonçalves, M. L. S.; Anal. Chim. Acta 1994, 284, 525.

13. Rocha, J. C.; Toscano, I. A. S.; Burba, P.; Talanta 1997, $44,69$.

14. Rosa, A. H.; Rocha, J. C.; Sargentini Jr, E.; European J. Soil Sci. 2000 (in press).

15. Dick, D.; Burba, P.; Herzog, H.; In: 9th International meeting of the International Humic Substances Society, Livro de resumos, 1998, p.75.

16. Whitehead, D. C.; Tinsley, J.; Soil Sci. 1967, 97, 34.

17. Jones, M. J.; Parsons, J. W.; J. Soil. Sci. 1972, 23, 119.

18. Stevenson, F. J.; Kidder, G.; Tilo, S. N.; Soil Sci. Soc. Amer. Proc. 1967, 31, 71.

19. Porter, L. K.; J. Agr. Food. Chem. 1967, 15, 807.

20. Clapp, C. E.; Hayes, M. H. B.; Swift, R. S. In: Organic substances in soil and water: Natural constituents and their influences on contaminant behaviour. Beck, A. J.; Jones, K. C.; Hayes, M. H. B.; Mingelgrin, U. Eds.; Royal Society of Chemistry; Cambridge 1993; p.31.

21. Parsons, J. W. In: Humic substances and their role in the environment. Frimmel, F. H. \& Christman, R. F. Eds.; John Wiley, Chichester 1988, p 03 - 14.

22. Rosa, A. H.; Rocha, J. C.; Furlan, M.; Zara, L. F.; Santos, A.; Anais Assoc. Bras. Quím. 1998, 47, 25.

23. Griffith, S. M.; Schnitzer, M.; Soil Sci. Amer. Proc. 1975, $39,861$.

24. Town, R. M.; Powell, H. K. J.; Anal. Chim. Acta 1992, 256, 81.

25. Peuravuori, J.; Pihlaja, K.; Anal. Chim. Acta 1997, 337, 133.

26. Swift, R. S.; In: Humic substances II: in search of structure. Hayes, M. H. B.; Maccarthy, P.; Malcolm, R. L.; Swift, R. S. (Eds.) Wiley; New York, 1989; p.450.

27. Kielland, J.; Amer. Chem. Soc. 1937, 59, 1675.

28. Atkins, P. W.; Physical Chemistry, $5^{\mathrm{a}}$ ed, Butler \& Tanner; Oxford 1994; p.841.

29. Schnitzer, M.; Khan, S. U.; Humic substances in the enviroment, Marcel Dekker; New York 1972.

30. Gascho, G. J.; Stevenson, F. J.; Soil Sci. Soc. Amer. Proc. 1968, 32, 117.

31. Zunic, W. M.; Ding, S.; Durig, J. R.; Cohen, A. D.; J. Mol. Struct. 1992, 287, 371.

32. Kononova, M. M.; Soil Organic Matter. Perghamon Press; Oxford 1966; p.75.

33. Pang, L. S. K.; Vassallo, A. M.; Wilson, M. A. Org. Geochem. 1990, 16, 853. 